\title{
How to Excite Nuclear Wavepackets into Electronically Degenerate States in Spin- Vibronic Quantum Dynamics Simulations
}

Pápai, Mátyás; Simmermacher, Mats; Penfold, Thomas James; Møller, Klaus Braagaard; Rozgonyi, Tamas

Published in:

Journal of Chemical Theory and Computation

Link to article, DOI:

$10.1021 /$ acs.jctc. 8 b00135

Publication date:

2018

Document Version

Peer reviewed version

Link back to DTU Orbit

Citation (APA):

Pápai, M., Simmermacher, M., Penfold, T. J., Møller, K. B., \& Rozgonyi, T. (2018). How to Excite Nuclear Wavepackets into Electronically Degenerate States in Spin-Vibronic Quantum Dynamics Simulations. Journal of Chemical Theory and Computation, 12(8), 3967-3974. https://doi.org/10.1021/acs.jctc.8b00135

\section{General rights}

Copyright and moral rights for the publications made accessible in the public portal are retained by the authors and/or other copyright owners and it is a condition of accessing publications that users recognise and abide by the legal requirements associated with these rights.

- Users may download and print one copy of any publication from the public portal for the purpose of private study or research.

- You may not further distribute the material or use it for any profit-making activity or commercial gain

- You may freely distribute the URL identifying the publication in the public portal 


\title{
How to Excite Nuclear Wavepackets into
}

Electronically Degenerate States in Spin-Vibronic Quantum Dynamics Simulations

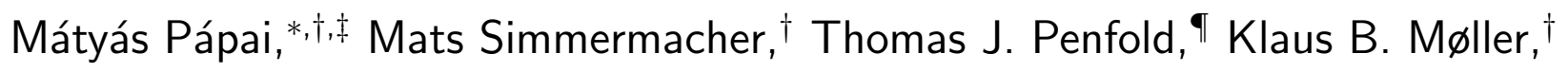 \\ and Tamás Rozgonyi*,§, \\ $\dagger$ †epartment of Chemistry, Technical University of Denmark, DK-2800, Kongens Lyngby, \\ Denmark \\ $\ddagger$ Wigner Research Centre for Physics, Hungarian Academy of Sciences, P.O. Box 49, \\ H-1525 Budapest, Hungary \\ \Chemistry - School of Natural and Environmental Science, Newcastle University, \\ Newcastle upon Tyne, NE1 7RU, United Kingdom \\ $\S$ Institute of Materials and Environmental Chemistry, Research Centre for Natural \\ Sciences, Hungarian Academy of Sciences, P.O. Box 286, H-1519 Budapest, Hungary \\ E-mail: papai@kemi.dtu.dk; rozgonyi.tamas@ttk.mta.hu
}




\begin{abstract}
The excited-state dynamics of two functional Fe-carbene complexes, $\left[\mathrm{Fe}(\mathrm{bmip})_{2}\right]^{2+}$ $\left(\right.$ bmip $=2,6$-bis $(3$-methyl-imidazole-1-ylidene)-pyridine $)$ and $\left[\mathrm{Fe}(\text { btbip })_{2}\right]^{2+}($ btbip $=$ 2,6-bis(3-tert-butyl-imidazole-1-ylidene)pyridine), are studied using the spin-vibronic model. In contrast to the usual projection of the ground state nuclear wavefunction onto an excited state surface, the dynamics are initiated by an explicit interaction term between the external time-dependent electric field (laser pulse) and the transition dipole moment of the molecule. The results show that the spin-vibronic model, as constructed directly from electronic structure calculations, exhibits erroneous, polarization-dependent relaxation dynamics stemming from artificial interference of coupled relaxation pathways. This is due to the lack of rotational invariance in the description of excitation into degenerate states. We introduce and discuss a correction using the spherical basis and complex transition dipole moments. This modification in the interaction Hamiltonian leads to rotationally invariant excitation and produces polarization-independent population dynamics.
\end{abstract}




\section{Introduction}

Understanding radiative and nonradiative decay mechanisms of photoexcited systems has important implications for our understanding of excited-state dynamics in fundamental as well as applied areas of research. ${ }^{1}$ Simultaneous progress in both theoretical and experimental approaches has made it possible to elucidate highly detailed information for complex systems. Many of these studies exploit a close synergy between experiment and theory, which is increasingly becoming a crucial component for achieving a deeper fundamental knowledge of ultrafast excited-state processes. ${ }^{2}$

Pump-probe techniques applying ultrashort (fs-ps) pulses have played a key role in resolving excited-state dynamics. To achieve an effective synergy, it is important that simulations replicate, as closely as required, the important experimental conditions. Fundamentally, this involves careful consideration of the accuracy of potential energy surfaces (PESs) and the nuclear motion on them. One may also be required to account for the effect of temperature and pressure or incorporate an accurate treatment of the environment, such as a solvent. An additional issue is the interaction between the molecule and the external time-dependent electric field of the laser pulse that generates the electronically excited state. Presently, the most common approach for initiating excited-state dynamics is the instantaneous projection of the nuclear wavefunction from the electronic ground state onto the excited-state potential energy surface. ${ }^{3-8}$ This approach assumes that the pump pulse prepares a well defined excited state. This is usually sufficient when considering smaller molecules with relatively low density of electronic states, i.e., the state excited can be clearly identified. However, for systems possessing high density of states, such as several classes of transition metal complexes, it might be necessary to account explicitly for the electronic excitation process. This is further complicated by the presence of states of different spin multiplicity. Although higher spin states such as triplets are not formally dipole coupled to the molecular ground state, the simultaneous presence of sizeable spin-orbit and nonadiabatic coupling means that mixing character between the states arises. This leads to a manifold of states that can be excited. 
The excited-state dynamics of transition metal complexes have been addressed using nonadiabatic molecular dynamics ${ }^{2,9,10}$ and quantum wavepacket approaches. ${ }^{6-8,10-13}$ For the latter, the simulations are performed within the basis of spin-vibronic model Hamiltonians. ${ }^{6-8}$ Since the full quantum dynamical treatment of the nuclear motion for relatively large (e.g., consisting of 40-60 atoms) molecular systems is computationally not feasible, these models include only the vibrational modes most relevant to the dynamics.

The spin-vibronic models, ${ }^{14}$ which are a generalized extension to the widely-adopted vibronic coupling Hamiltonian, ${ }^{15}$ are powerful for deciphering the early time photophysics and to discover the subtle effects driving it. Despite its advantages, the important challenge of pushing the methodology towards more realistic simulations still remains. In the present work, we use two prototypical Fe- $N$-heterocyclic carbene (NHC) complexes for ultrafast excited-state dynamics, $\left[\mathrm{Fe}(\text { btbip })_{2}\right]^{2+}($ btbip $=2,6$-bis(3-tert-butyl-imidazole-1ylidene)pyridine) $(\mathbf{1})$ and $\left[\mathrm{Fe}(\mathrm{bmip})_{2}\right]^{2+}(\mathbf{2})$ (bmip $=2,6$-bis(3-methyl-imidazole-1-ylidene) pyridine) (Figure 1, top), ${ }^{16,17}$ to demonstrate that the application of spin-vibronic Hamiltonians constructed directly from quantum chemical data, using the standard electron-field interaction term, leads to unphysical polarization-dependent dynamics. We present a correction in the interaction Hamiltonian, which avoids this problem and correctly describes the excitation to, and hence, subsequent photorelaxation processes from electronically degenerate states.

\section{Theoretical Details}

Herein, we start from the spin-vibronic models recently developed and described in refs. 12 and 8 for $\left[\mathrm{Fe}(\text { btbip })_{2}\right]^{2+}$ and $\left[\mathrm{Fe}(\text { bmip })_{2}\right]^{2+}$, respectively. These contain the four most decisive normal mode degrees of freedom for both complexes: tuning modes driving the largest nuclear motion and coupling modes responsible for the strongest nonadiabatic couplings (NACs) between different electronic states. In the models, the singlet and triplet excited 

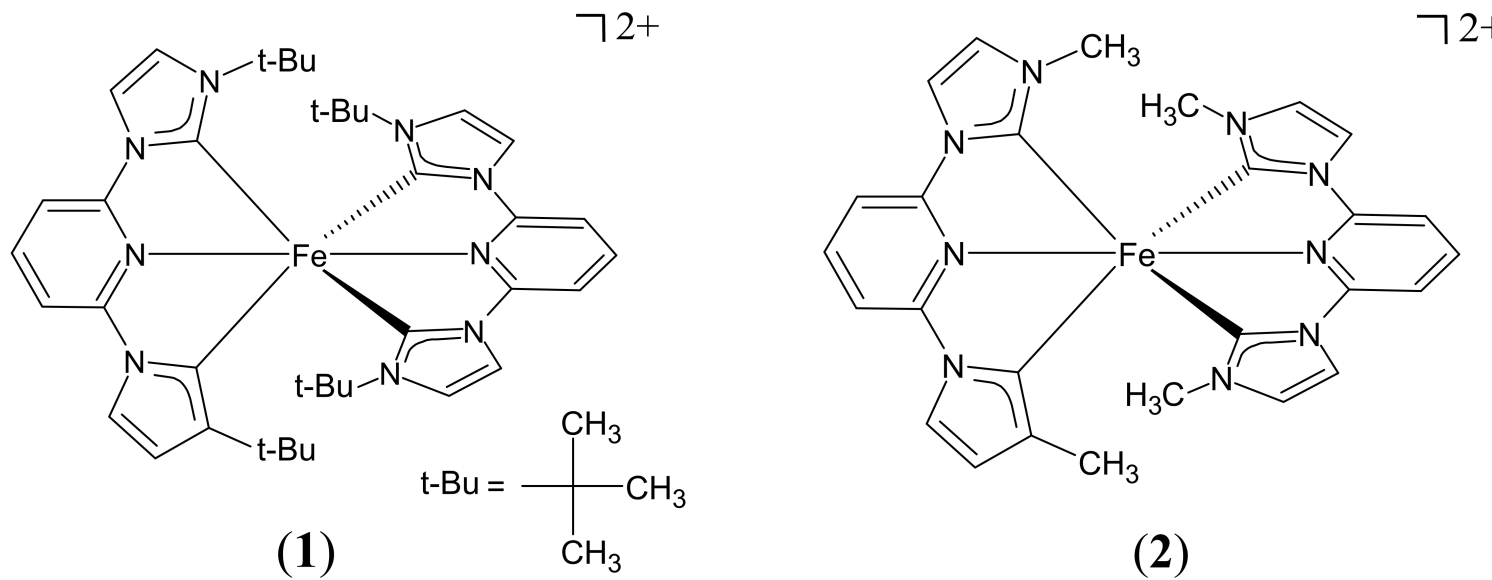

(2)
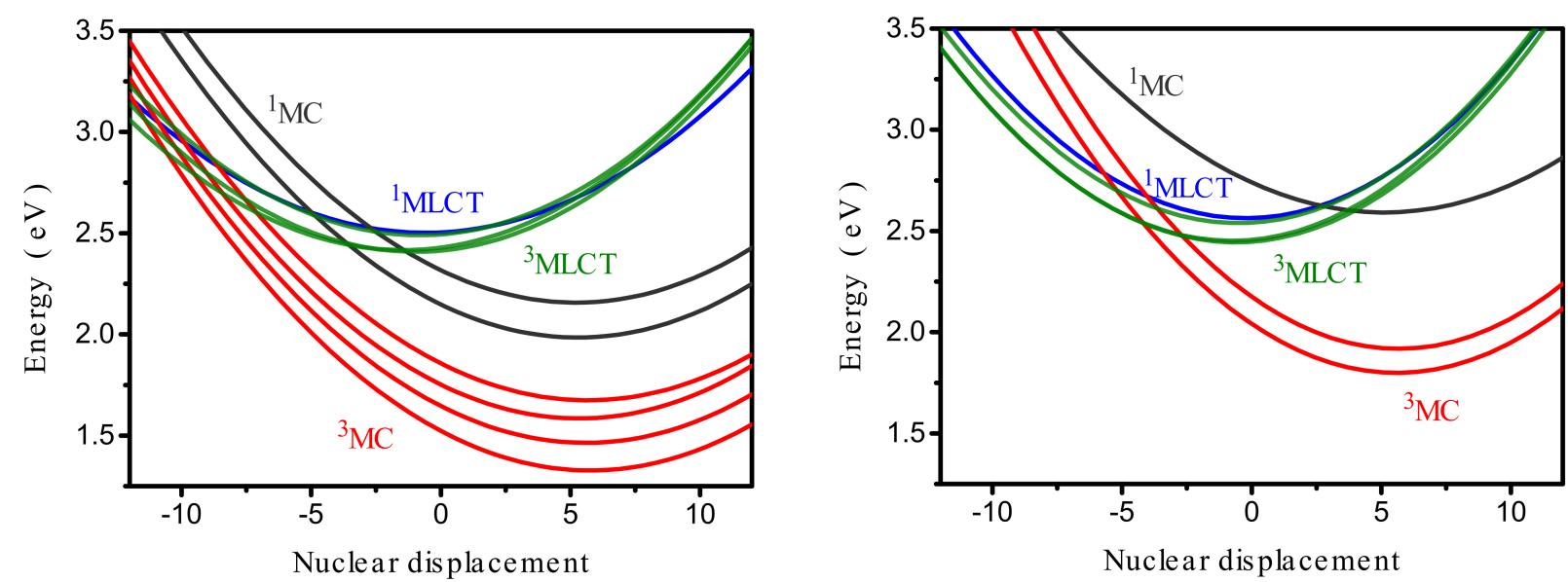

Figure 1: Molecular structure (top) and excited-state potential energy curves (bottom) along the dominant (lowest-frequency breathing) mode of the investigated $\left[\mathrm{Fe}(\mathrm{btbip})_{2}\right]^{2+}(\mathbf{1})$ and $\left[\mathrm{Fe}(\mathrm{bmip})_{2}\right]^{2+}(\mathbf{2})$ complexes. The lines represent diabatic potentials obtained from fits to adiabatic energies calculated by time-dependent density functional theory (TD-DFT), yielding the diabatic vibronic coupling Hamiltonian. ${ }^{8,12}$ Nuclear displacements are given in dimensionless mass-frequency weighted normal coordinates.

states included were those that are energetically accessible from the lowest optically bright singlet metal-to-ligand charge transfer $\left({ }^{1} \mathrm{MLCT}\right)$ states. These include two ${ }^{1} \mathrm{MLCT}$, three ${ }^{1} \mathrm{MC}(\mathrm{MC}=$ metal-centered, i.e., arising from $d$ - $d$ excitations $)$, four ${ }^{3} \mathrm{MLCT}$ and six ${ }^{3} \mathrm{MC}$ states for $\left[\mathrm{Fe}(\text { btbip })_{2}\right]^{2+}$, and two ${ }^{1} \mathrm{MLCT}$, two ${ }^{1} \mathrm{MC}$, four ${ }^{3} \mathrm{MLCT}$ and three ${ }^{3} \mathrm{MC}$ states for $\left[\mathrm{Fe}(\mathrm{bmip})_{2}\right]^{2+}$. Accounting for the three different values of the $M_{s}$ quantum number $(-1,0,1)$ for each triplet state, these lead altogether to 36 and 26 electronic states for $\left[\mathrm{Fe}(\mathrm{btbip})_{2}\right]^{2+}$ and $\left[\mathrm{Fe}(\mathrm{bmip})_{2}\right]^{2+}$, respectively. Figure 1 depicts the diabatic excited-state potential energy curves of the two carbene complexes along their lowest-frequency breathing modes, identified 
as the dominant vibrational modes for the photorelaxation cascade.

These models described in refs. 8 and 12 are extended in the present work by the inclusion of an explicit description of the interaction between the molecule and the time-dependent electric field of the exciting laser pulse. The applied spin-vibronic Hamiltonian operator is then expressed as

$$
\boldsymbol{H}(t)=\left(T_{N}+V_{0}\right) \mathbf{1}+\boldsymbol{W}+\boldsymbol{S}+\boldsymbol{\mu}_{\mathbf{0}} \varepsilon E(t)
$$

where $T_{N}$ is the kinetic energy operator, $V_{0}$ is the ground-state (harmonic) potential, $\mathbf{1}$ is the unit matrix, $\boldsymbol{W}$ expresses the vibronic coupling, and $\boldsymbol{S}$ represents the spin-orbit coupling (SOC) matrix. The last term in Equation 1 describes the interaction of the molecule with the time-dependent electric field, $E(t)$, of a linearly polarized pump laser pulse in the semiclassical dipole approximation, with $\boldsymbol{\mu}_{\mathbf{0}}$ being the zeroth-order term of a Taylor expansion of the transition dipole moment (TDM) $\boldsymbol{\mu}(q)$, where $q$ is the normal mode coordinate, around the Franck-Condon point. $\varepsilon$ is the polarization vector of the laser pulse. The laser frequencies are chosen to be resonant for the optically bright ${ }^{1}$ MLCT states. The pulses are transform-limited and have a Gaussian intensity profile with 60 fs full width at half maximum. The peak of the pulse is centered at $120 \mathrm{fs}$ and the intensity is chosen small enough to avoid the saturation of absorption. The $\boldsymbol{S}$ matrix and the relevant elements of $\boldsymbol{\mu}_{\mathbf{0}}$ are determined at the Franck-Condon (FC) geometry. ${ }^{18}$ The first order derivatives of the TDMs with respect to the nuclear displacements along the normal modes are found to be negligible compared to the corresponding zeroth-order terms computed at the FC point $\left(\boldsymbol{\mu}_{\mathbf{0}}\right)$. Therefore, only the elements of the $\boldsymbol{\mu}_{\mathbf{0}}$ matrix are included in the Hamiltonian. The off-diagonal elements of the potential coupling matrix, $\boldsymbol{W}$, between electronic states $i$ and $j$ can be expressed as: 


$$
W_{i j}=\sum_{\nu} \lambda_{i j}^{(\nu)} q_{\nu}
$$

where $q_{\nu}$ is the dimensionless, mass-frequency weighted normal coordinate of vibrational mode $\nu$, and $\lambda_{i j}^{(\nu)}$ is the linear nonadiabatic coupling constant. The $\boldsymbol{W}$ matrix contains the energies of the electronic states $\left(W_{i}^{(0)}\right.$ for state $\left.i\right)$ at the Franck-Condon geometry, as well as first and second-order on-diagonal elements, which are related to the forces acting on the excited-state potentials, and the force constants, respectively:

$$
W_{i i}=W_{i}^{(0)}+\sum_{\nu} \kappa_{i}^{(\nu)} q_{\nu}+\frac{1}{2} \sum_{\nu} \gamma_{i}^{(\nu)} q_{\nu}^{2}
$$

The determined $\lambda_{i j}^{(\nu)}, \kappa_{i}^{(\nu)}$, and $\gamma_{i}^{(\nu)}$ coefficients are those, which, by the diagonalization of the $\boldsymbol{W}$ matrix, lead to adiabatic potential energy surfaces in the best agreement with those computed by electronic structure calculations, in the present case, TD-DFT (TDB3LYP*19). The application of this approach, known as diabatization by ansatz, ${ }^{14,20}$ leads to the generation of a set of coupled diabatic states. Note that the TDMs and SOCs do not need to be diabatized, as they are only computed at the FC geometry, at which the adiabatic and diabatic representations are chosen to be identical. The matrix elements of $\boldsymbol{W}$ and $\boldsymbol{S}$ are given in refs. 8 and 12 . The transition dipole moments are computed by the same TD-DFT method, as the one used to calculate the excited-state potentials. ${ }^{8,12}$

In this work, we consider both molecules to have their principal $\mathrm{C}_{2}(z)$ symmetry axis aligned along the laboratory $z$-axis, and therefore the planes of the two ligands in both molecules lie in the $x z$ and $y z$-planes. Both complexes possess $\mathrm{D}_{2 \mathrm{~d}}$ equilibrium point group symmetry, in which the $\left(\boldsymbol{\mu}_{\boldsymbol{n}, \boldsymbol{x}}, \boldsymbol{\mu}_{\boldsymbol{n}, \boldsymbol{y}}\right)$ TDMs of the $n \epsilon\left\{{ }^{1} \mathrm{MLCT},{ }^{1} \mathrm{MC}\right\}$ states for a given $n$ transform together as the twofold-degenerate E irreducible representation, while a transition moment in the $z$-direction, $\boldsymbol{\mu}_{\boldsymbol{z}}$, would transform as $\mathrm{B}_{2}$. In both molecules, the optically 
bright ${ }^{1} \mathrm{MLCT}$, as well as the energetically closest ${ }^{1} \mathrm{MC}$ states are degenerate. This is to say that they both show E symmetry and the corresponding TDM vectors $\boldsymbol{\mu}_{\boldsymbol{n}, \boldsymbol{x}}$ and $\boldsymbol{\mu}_{\boldsymbol{n}, \boldsymbol{y}}$ are orthogonal, lying in the $x y$-plane, on the axes, as shown in Figure 2 for $\left[\mathrm{Fe}(\mathrm{btbip})_{2}\right]^{2+} \cdot{ }^{21}$ Note that although the length of the transition moment vectors to the ${ }^{1} \mathrm{MC}$ states is comparable of those the ${ }^{1}$ MLCTs, the ground-state population is mainly $(\sim 90 \%)$ excited to the optically bright ${ }^{1}$ MLCTs, as the laser frequency is chosen to be resonant for these states. Therefore, we henceforth focus on the excitation into the degenerate ${ }^{1}$ MLCT manifold. However, we stress that that the excitation into ${ }^{1} \mathrm{MC}$ states is incorporated in the simulations, and the correct description of the electronic excitation into degenerate states is independent of the applied laser frequency. The TDMs of $\left[\mathrm{Fe}(\mathrm{bmip})_{2}\right]^{2+}$ are analogous to those shown in Figure 2 only that the length of the TDM vectors to the ${ }^{1} \mathrm{MC}$ states is reduced by a factor of ca. 2. The $\boldsymbol{\mu}_{\boldsymbol{z}}$ transition moment is irrelevant for the description of excitation from the ground state into doubly-degenerate states, as it vanishes for any transition from a totally symmetric electronic state into the degenerate manifold. Consequently, in the present work, the polarization of the electric field is considered to lie in the $x y$-plane and is defined throughout by the $\varphi$ angle between the $\varepsilon$ polarization vector and the $x$-axis, see the inset of Figure 2 .

The quantum dynamics simulations are performed using the Multiconfigurational TimeDependent Hartree (MCTDH) method. ${ }^{22-24}$ The initial nuclear wavepacket is built from eigenfunctions of the ground state harmonic oscillator. The sizes of the basis sets applied in the simulations are taken from refs. 8,12 (except that the number of single particle functions for the ground state is increased from 1 to 10 , as the simulations are initiated from this state, not from the photoexcited ${ }^{1}$ MLCT state ${ }^{8,12}$ ), which ensures convergence for the full duration of the simulations. 


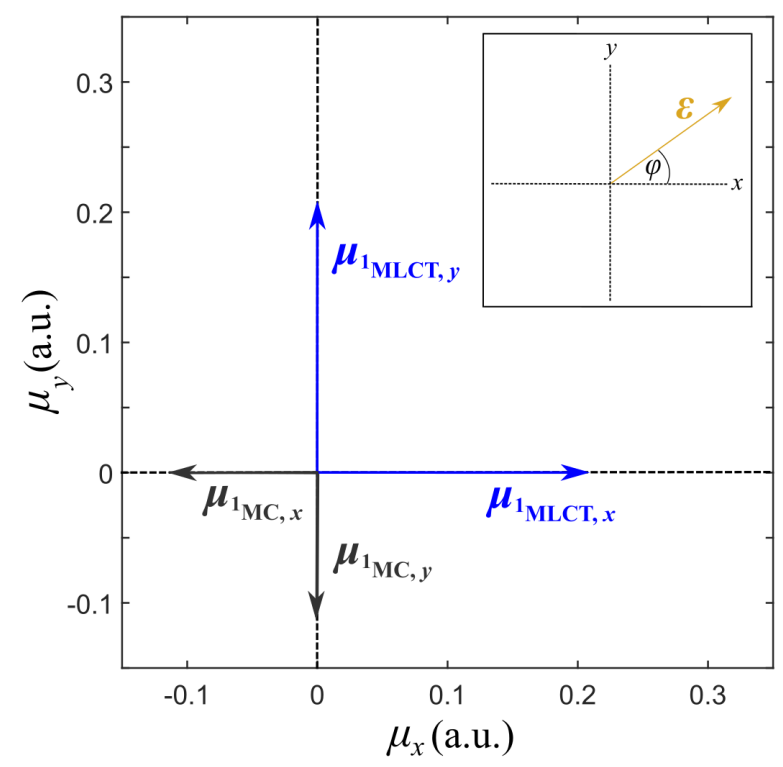

Figure 2: Graphical representation of the TD-DFT-computed $\boldsymbol{\mu}_{\boldsymbol{n}, \boldsymbol{x}}, \boldsymbol{\mu}_{\boldsymbol{n}, \boldsymbol{y}}$ transition dipole moments corresponding to the excitation from the ground state to the degenerate pair of $n \epsilon$ $\left\{{ }^{1} \mathrm{MLCT},{ }^{1} \mathrm{MC}\right\}$ states of $\left[\mathrm{Fe}(\text { btbip })_{2}\right]^{2+}$. Note that the choice of these TDMs is not unique, since degenerate states can be freely rotated among themselves in the Hilbert space. Inset: $\varphi$ is defined as the angle between the $\varepsilon$ polarization vector of the pump pulse and the $x$-axis.

\section{Results and Discussion}

Figures 3 and 4 present the excited-state population kinetics of $\left[\mathrm{Fe}(\mathrm{btbip})_{2}\right]^{2+}$ and $\left[\mathrm{Fe}(\text { bmip })_{2}\right]^{2+}$, respectively, following excitations at four polarization angles: $\varphi=\left\{-45^{\circ}, 0^{\circ}\right.$, $\left.45^{\circ}, 90^{\circ}\right\}$. This shows that for $\left[\mathrm{Fe}(\text { btbip })_{2}\right]^{2+}$, the obtained population dynamics are highlydependent on the polarization; ${ }^{25}$ the largest difference, reflected in the rate of ${ }^{1} \mathrm{MLCT}$ decay and ${ }^{1} \mathrm{MC} /{ }^{3} \mathrm{MC}$ growth, is observed for $\varphi=45^{\circ}$ and $-45^{\circ}$. For $\left[\mathrm{Fe}(\mathrm{bmip})_{2}\right]^{2+}$, the effect of the polarization is significantly weaker.

The polarization-dependent dynamics presented in Figure 3 are inconsistent with fundamental molecular symmetry considerations for degenerate excited states. Namely, the pair of degenerate states transforming as the same $\mathrm{E}\left(\mathrm{D}_{2 \mathrm{~d}}\right)$ irreducible representation, such as ${ }^{1} \mathrm{MLCT}_{1}$ and ${ }^{1} \mathrm{MLCT}_{2}$, and thus, the corresponding transition dipole moments, cannot be uniquely defined. This means that any linear combination of the pair of degenerate states can be chosen, leading to rotation of the TDMs in the $x y$-plane and identical transitions to the 

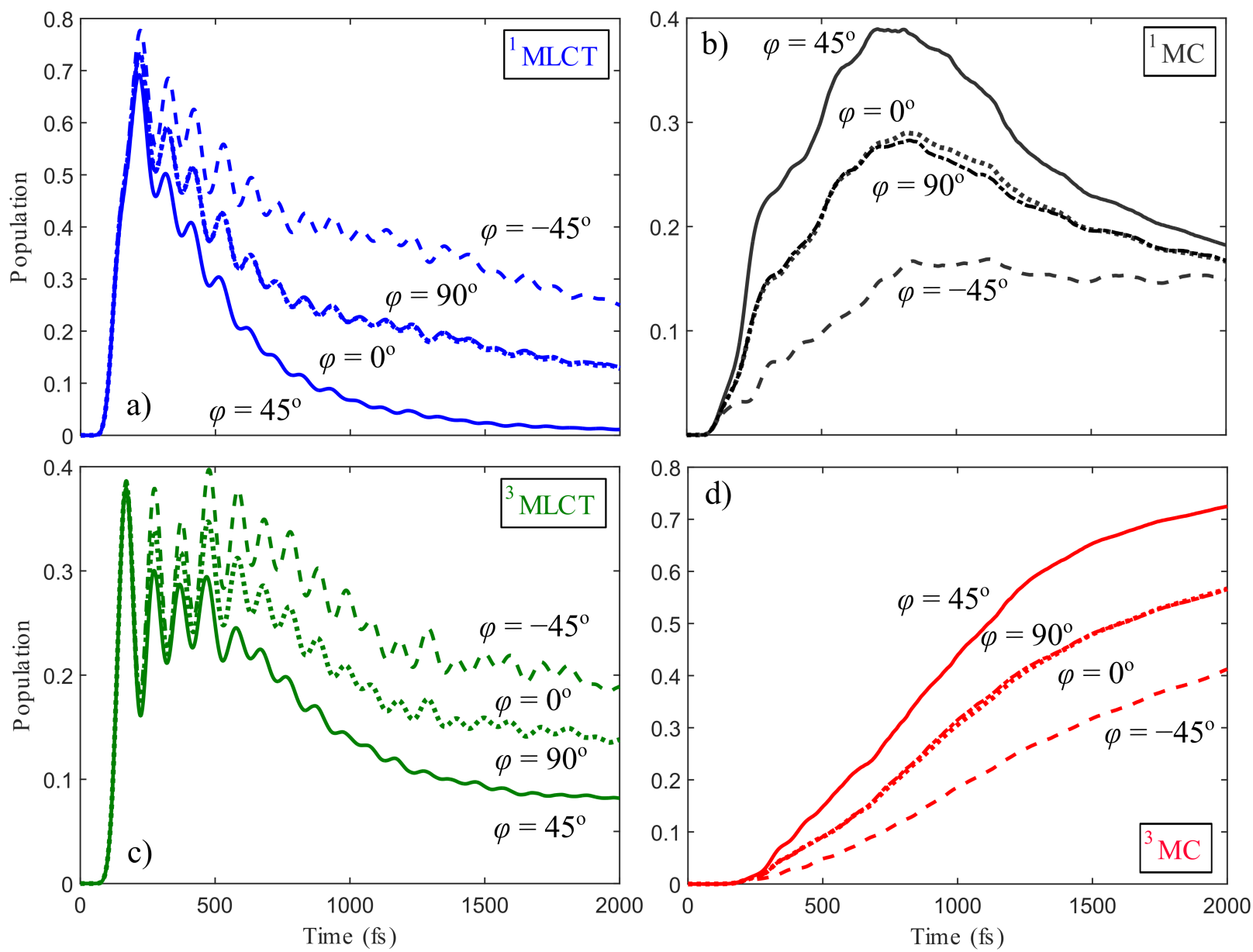

Figure 3: Diabatic population dynamics for $\left[\mathrm{Fe}(\text { btbip })_{2}\right]^{2+}$, for four polarization angles, normalized to the total excited state populations. As is seen from the panels, polarization dependence is most pronounced for the ${ }^{1} \mathrm{MLCT}$ and ${ }^{1} \mathrm{MC}$ states during the first ps.

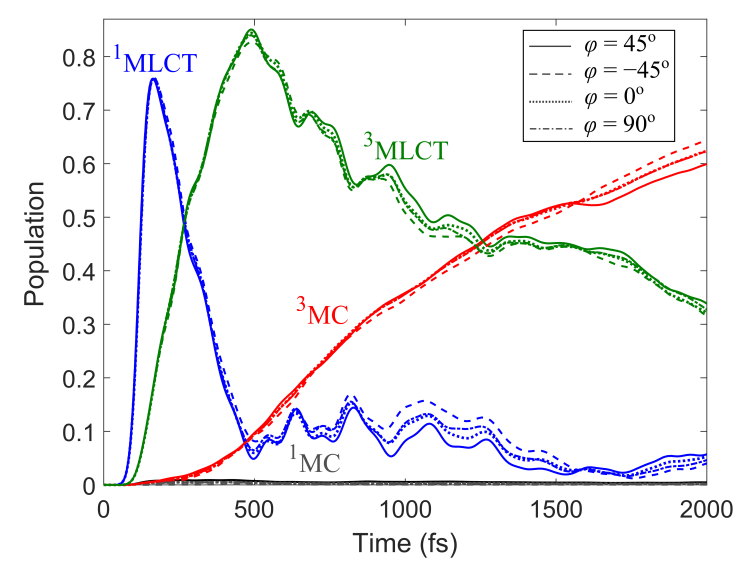

Figure 4: Diabatic population dynamics for $\left[\mathrm{Fe}(\mathrm{bmip})_{2}\right]^{2+}$, for four different polarization angles, normalized to the total excited state populations. As is clear from the figure, the difference between the corresponding population curves is small. 

and rotating the polarization vector by the same angle, which must not affect the simulated dynamics the way it is seen in Figure 3. As we will discuss later in this section, this inconsistency derives from a lack of rotational invariance of the computational description of the excitation process, which has to be corrected to produce polarization-independent population dynamics.

Figure 2 shows that in cases of $\varphi=45^{\circ}$ and $\varphi=-45^{\circ}$ both ${ }^{1}$ MLCTs are excited (in the former case with the same and in the latter with opposite signs), which creates a propensity for interference effects between the coupled relaxation pathways. Indeed, the polarizationdependent dynamics observed in Figure 3 for $\left[\mathrm{Fe}(\text { btbip })_{2}\right]^{2+}$ is consistent with interferences between the internal conversion relaxation pathways from the ${ }^{1} \mathrm{MLCT}_{1}$ and ${ }^{1} \mathrm{MLCT}_{2}$ states to the same ${ }^{1} \mathrm{MC}$ state. This occurs due to the nonadiabatic coupling between the pair of degen-

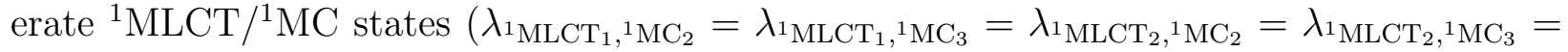
$\lambda_{{ }^{1} \mathrm{MLCT},{ }^{1} \mathrm{MC}}$, see Figure 5a) and the fact that both ${ }^{1} \mathrm{MLCT}$ states are excited. Figure 4 reveals that the situation is quite different for $\left[\mathrm{Fe}(\mathrm{bmip})_{2}\right]^{2+}$ : only a small polarization dependence arises in the simulated population dynamics. This is because the ${ }^{1} \mathrm{MC}$ states of this complex are energetically unaccessible from the ${ }^{1} \mathrm{MLCT}$, as is clear from Figure 1. Consequently, only a single dominant ${ }^{1} \mathrm{MLCT} \rightarrow{ }^{3} \mathrm{MLCT} \rightarrow{ }^{3} \mathrm{MC}$ pathway is observed. Importantly, the ${ }^{1} \mathrm{MLCT} \rightarrow{ }^{3} \mathrm{MLCT}$ pathway involves decay of the two initially excited ${ }^{1} \mathrm{MLCTs}$ through separate intersystem crossing relaxation channels to the highest-lying ${ }^{3} \mathrm{MLCT}$ state, ${ }^{3} \mathrm{MLCT}_{4}$, as illustrated in Figure 5c. Thus, no ${ }^{1} \mathrm{MLCT}-{ }^{1} \mathrm{MC}$ interferences can rise here. The small, nonvanishing effect is attributed to interferences occurring through the nonadiabatic coupling between the degenerate pairs of ${ }^{3} \mathrm{MLCT}$ and ${ }^{3} \mathrm{MC}$ states, analogous to the one illustrated in Figure 5a for ${ }^{1} \mathrm{MLCT}$ and ${ }^{1} \mathrm{MC}$ states. The contribution of this relaxation channel to the full ${ }^{3} \mathrm{MLCT} \rightarrow{ }^{3} \mathrm{MC}$ population transfer is, however, rather small. This explains the much weaker polarization dependence in the resulting population dynamics than the one caused by the ${ }^{1} \mathrm{MLCT}-{ }^{1} \mathrm{MC}$ relaxation pathway in $\left[\mathrm{Fe}(\text { btbip })_{2}\right]^{2+}$. 
a) Nonadiabatic coupling (NAC)

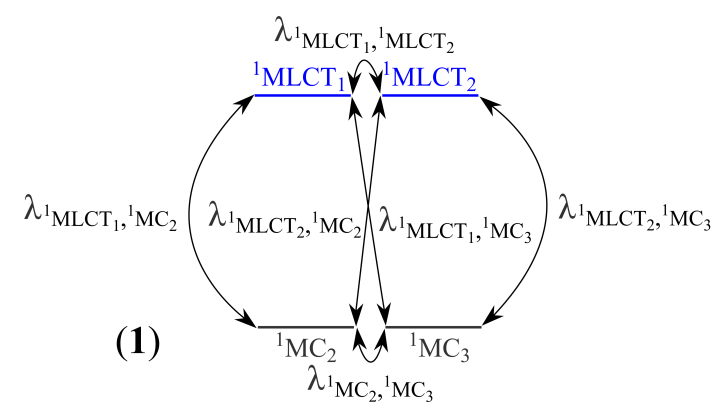

b) Spin-orbit coupling (SOC)

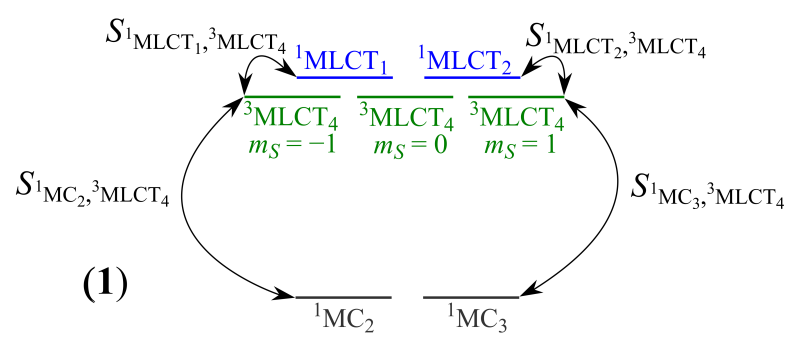

c) Spin-orbit coupling (SOC)

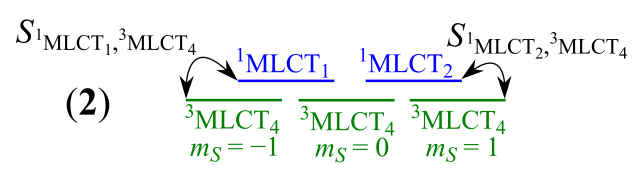

Figure 5: Schematic illustration of the two possible coupling mechanisms determining the initial excited-state dynamics of the two investigated carbene complexes: a) singlet-singlet nonadiabatic coupling for $\left[\mathrm{Fe}(\text { btbip })_{2}\right]^{2+}(\mathbf{1})$ and b), c) singlet-triplet spin-orbit coupling for $\left[\mathrm{Fe}(\text { btbip })_{2}\right]^{2+}$ and $\left[\mathrm{Fe}(\text { bmip })_{2}\right]^{2+}(\mathbf{2})$, respectively. For $\left[\mathrm{Fe}(\text { bmip })_{2}\right]^{2+}$, only SOC is relevant during the first few hundred femtoseconds. For $\left[\mathrm{Fe}(\text { btbip })_{2}\right]^{2+}$, both coupling mechanisms are operative. Note that these coupling schemes are consistent with the pair of degenerate singlet states, whose TDM vectors $\boldsymbol{\mu}_{\boldsymbol{n}, \boldsymbol{x}}$ and $\boldsymbol{\mu}_{\boldsymbol{n}, \boldsymbol{y}}$ lie exactly on the $x$ and $y$ axes, as illustrated in Figure 2. 
To further investigate the nature of the observed interferences, we have created a simplified model Hamiltonian for $\left[\mathrm{Fe}(\mathrm{btbip})_{2}\right]^{2+}$, in which all spin-orbit couplings and, therefore, triplet states were neglected. Excitation in this case was treated in the impulsive limit, i.e., the initial wavepacket was constructed by projecting the ground state vibrational wavefunction onto the two ${ }^{1} \mathrm{MLCT}$ states with different amplitudes. Here, the different laser polarizations were simulated by different weights $\left(w_{1}, w_{2}\right)$ to scale the initial wavefunction in the two degenerate ${ }^{1}$ MLCT states. Figure 6 presents the results of three simulations, in which the relative weights of $\left(+1 / \sqrt{2},+1 / \sqrt{2}\right.$, in-phase, i.e., $\left.\varphi=45^{\circ}\right),\left(+1,0\right.$, i.e., $\left.\varphi=0^{\circ}\right)$, and $\left(-1 / \sqrt{2},+1 / \sqrt{2}\right.$, out-phase, i.e., $\left.\varphi=-45^{\circ}\right)$ were used. These simulations illustrate the cases of constructive $(+1 / \sqrt{2},+1 / \sqrt{2})$, vanishing $(1,0)$ and destructive $(+1 / \sqrt{2},-1 / \sqrt{2})$ interference for the coupled ${ }^{1} \mathrm{MLCT} \rightarrow{ }^{1} \mathrm{MC}$ decay pathways. The observed effect is analogous for the full model, with the only difference being that a fraction of the excited-state population in the full model is additionally converted from the ${ }^{1} \mathrm{MLCT}$ to the ${ }^{1} \mathrm{MC}$ via the ${ }^{3}$ MLCT states. This additional relaxation channel, which is not observed in the absence of SOC, as shown in Figure 6, is however not influenced by the laser polarization.

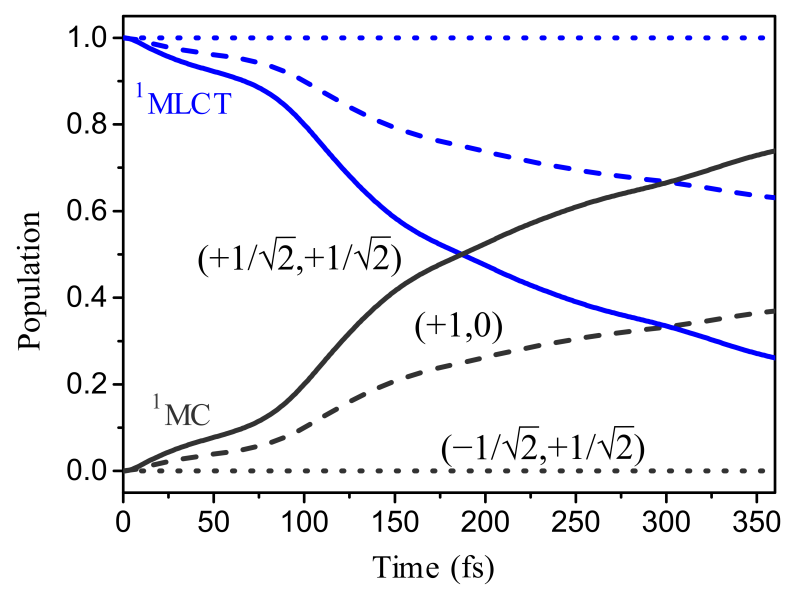

Figure 6: Population dynamics from simulations using the singlet states of $\left[\mathrm{Fe}(\mathrm{btbip})_{2}\right]^{2+}$ only with three initial conditions: Solid and dotted curves are obtained by launching wavepackets on the two ${ }^{1}$ MLCT states with the same (constructive interference) and opposite (destructive interference) phases, respectively, while dashed curves show results obtained by launching the wavepacket on only the ${ }^{1} \mathrm{MLCT}_{1}$ state (no interference). Note that results obtained with the $(0,1)$ relative weights are identical to those of $(1,0)$ weights. 
In the following, we analyze the origin of the artificial interferences and develop a correction for this erroneous behavior.

In the so far presented simulations, the $\boldsymbol{\mu}_{\boldsymbol{n}, \boldsymbol{x}}$ and $\boldsymbol{\mu}_{\boldsymbol{n}, \boldsymbol{y}}\left(n \in\left\{{ }^{1} \mathrm{MLCT},{ }^{1} \mathrm{MC}\right\}\right)$ transition dipole moment vectors, shown in Figure 2, have been treated separately and were therefore uniquely defined. This is, however, unphysical, since the two vectors for a given $n$ should refer to indistinguishable degenerate states, and transform together as the twofold-degenerate E irreducible representation of the $\mathrm{D}_{2 \mathrm{~d}}$ point group. The description of the excitation process as two separate transitions translates into the above reported artificial interference of relaxation pathways through the nonadiabatic coupling. This becomes clear from the following.

In the simulations, the electronic excitation is described by the $\boldsymbol{\mu}_{\mathbf{0}} \varepsilon E(t)$ interaction term (see Equation 1). With $\boldsymbol{\mu}_{1_{\text {MLCT, } \boldsymbol{x}}}=(\mu, 0), \boldsymbol{\mu}_{\mathbf{1}_{\mathbf{M L C T}, \boldsymbol{y}}}=(0, \mu)$, where $\mu$ is the TDM computed by TD-DFT (see Figure 2), and $\varepsilon=(\cos \varphi, \sin \varphi)$, the interaction terms for transitions from the ground state to the ${ }^{1} \mathrm{MLCT}_{1}$ and ${ }^{1} \mathrm{MLCT}_{2}$ states will be $\mu E(t) \cos \varphi$ and $\mu E(t) \sin \varphi$, respectively. As shown in Figure 5a, each of these ${ }^{1}$ MLCT states is coupled to both components of the degenerate ${ }^{1} \mathrm{MC}$ manifold, i.e. ${ }^{1} \mathrm{MC}_{2}$ and ${ }^{1} \mathrm{MC}_{3}$, with the same coupling strength, $\lambda_{{ }^{1} \mathrm{MLCT},{ }_{\mathrm{MC}}}=\lambda$. Therefore, the probability of the transition from the ground state to the ${ }^{1} \mathrm{MC}$ degenerate manifold via the two degenerate ${ }^{1}$ MLCT states is proportional to:

$$
|\lambda \mu E(t) \cos \varphi+\lambda \mu E(t) \sin \varphi|^{2}=|\lambda \mu E(t)|^{2}(1+\sin 2 \varphi)
$$

The appearing unphysical angle dependence thus originates from the incorrect, rotationalvariant description of the excitation into degenerate states, which is reflected in the observed interferences and polarization-dependent dynamics. We note that this problem is equally present in the case of treating the excitation in the impulsive limit, i.e., just projecting the wavepacket onto the excited-state surfaces with the weights reflecting the laser polarization, as is clear from Figure 6.

The problem of this unphysical polarization dependence is solved by ensuring the cor- 
rect symmetry transformation of the excitation process. In the case of excitation from the electronic ground state into degenerate states, the direct product of the irreducible representations of these states in the $\mathrm{D}_{2 \mathrm{~d}}$ point group is $\mathrm{A}_{1} \otimes \mathrm{E}=\mathrm{E}$. This determines that the dipole moment operator has to transform as $\mathrm{E}$, in order to obtain nonvanishing transition matrix elements for the $\mathrm{A}_{1} \rightarrow \mathrm{E}$ transition. This has the consequence that the $\left(\boldsymbol{\mu}_{x}, \boldsymbol{\mu}_{\boldsymbol{y}}\right)$ transition dipole moments have to transform together and thus these TDMs cannot be represented in one dimension in real space. However, such a one-dimensional representation is possible in complex space using the spherical basis. ${ }^{26,27}$ Therefore, we rewrite the TDM vectors as:

$$
\boldsymbol{\mu}_{n, x}^{\prime}=\mu_{n, x}, \quad \mu_{n, y}^{\prime}=i \cdot \mu_{n, y}
$$

where $i$ is the imaginary unit. We mention that this solution is closely related to the $E \times e$ Jahn-Teller Hamiltonian of Köppel et al., ${ }^{15}$ transformed to a complex eletronic and vibrational basis. This ensures that the vibronic angular momentum is diagonal and commutes with the Hamiltonian, eliminating any artificial rotational variance.

Crucially, using Equaton 5, the overall transition probability from the ground to the degenerate ${ }^{1} \mathrm{MC}$ states via internal conversion through the ${ }^{1} \mathrm{MLCT}$ states becomes proportional to:

$$
|\lambda \mu E(t) \cos \varphi+i \cdot \lambda \mu E(t) \sin \varphi|^{2}=|\lambda \mu E(t)|^{2} .
$$

Equation 6 shows correctly no angular dependence and thus ensures rotational invariance. This description is transferable to the case of impulsive excitation by weighting the initial nuclear wavefunction in the excited states with $(+1 / \sqrt{2},+i / \sqrt{2}),(+1,0)$, and $(-1 / \sqrt{2},+i / \sqrt{2})$, as well as to any molecule, in which excitation occurs to degenerate E states.

We have repeated the excited-state simulations for both complexes utilizing the TDM vectors defined in Equation 5, while keeping all other matrix elements unchanged. The results of these simulations are shown in Figures 7 and 8 for $\left[\mathrm{Fe}(\mathrm{btbip})_{2}\right]^{2+}$ and $\left[\mathrm{Fe}(\mathrm{bmip})_{2}\right]^{2+}$, respectively. It is apparent from these figures that the application of complex TDMs leads 
to population dynamics that are same for all four polarization angles (in fact, this holds for any value of $\varphi$ ). The reason is that the complex representation of TDMs ensures rotationally invariant excitation into degenerate states and thus the correct phases of the nuclear wavefunctions, which eliminates the unphysical interferences of relaxation pathways. Therefore, all polarizations yield identical dynamics, which for $\left[\mathrm{Fe}(\text { btbip })_{2}\right]^{2+}$, essentially coincide with those obtained without applying the correction in the interaction Hamiltonian, for $\varphi=\left\{0^{\circ}, 90^{\circ}\right\}$, shown in Figure 3. Namely, the latter two polarizations account correctly for the fact that the transition occurs in the direction of the electric field. However, this is clearly not the case for any other polarization angle. For them, correct dynamics are obtained only if the correction introduced in this section is applied.

\section{Conclusion}

In this work, we have shown on two functional Fe-carbene complexes that spin-vibronic Hamiltonians created directly from ab initio data, using the standard electron-field interaction term, can lead erroneously to polarization-dependent excited-state dynamics. This problem arises due to the incorrect description of excitation of nuclear wavepackets into electronically degenerate states, resulting in artificial interference of coupled relaxation pathways. The reason for this deficiency is that the simulated excitation process lacks invariance with respect to rotation in the $x y$-plane. This translates into unphysical polarization-dependent dynamics through the nonadiabatic couplings among pairs of degenerate states. Crucially, the complex representation of transition dipole moments ensures rotational invariance. The correct description of the excitation process eliminates the interferences and thus produces correct excited-state dynamics for both investigated complexes. An equivalent treatment is necessary for molecules, in which photorelaxation occurs from degenerate E excited states. 

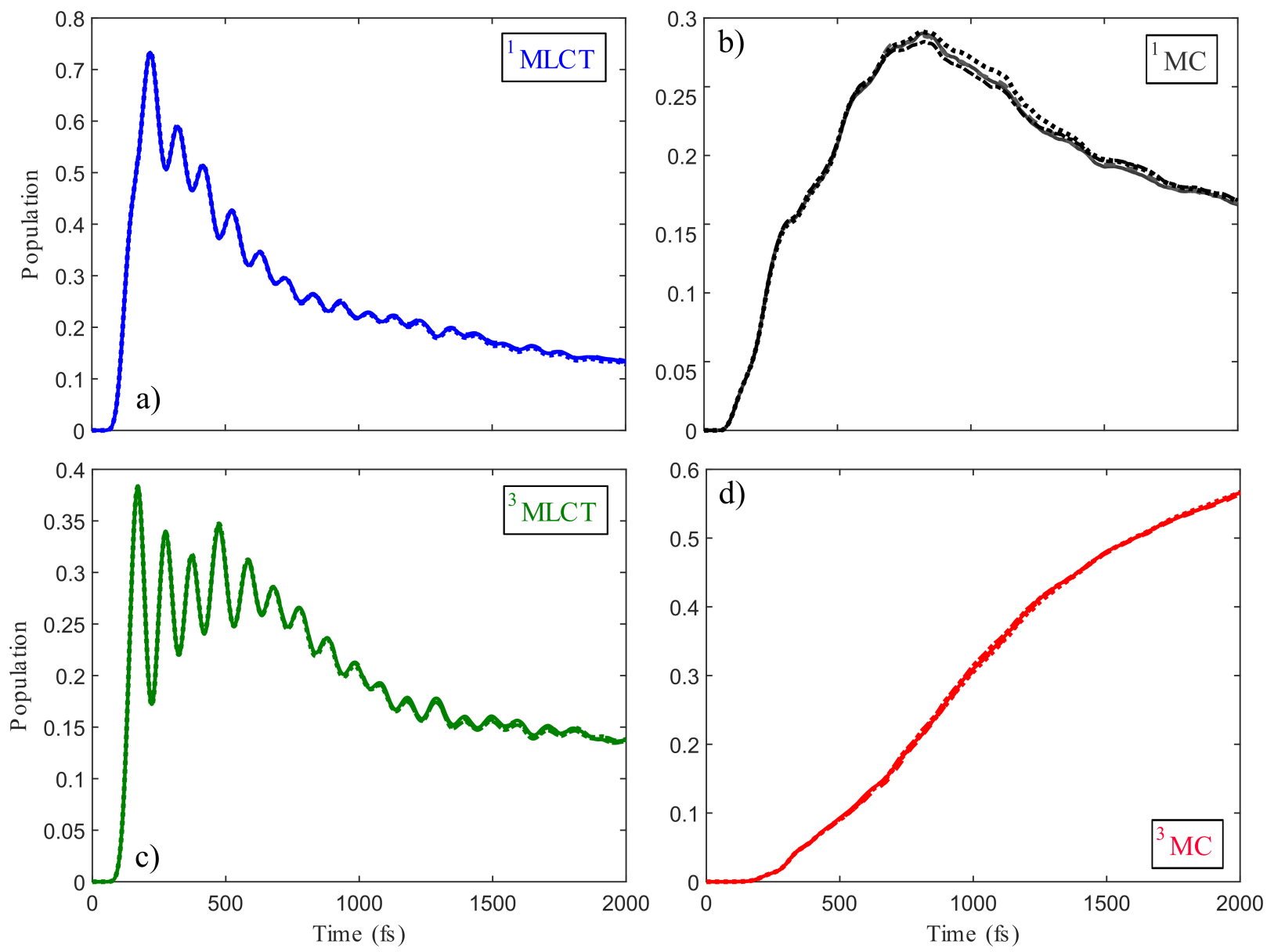

Figure 7: Diabatic population dynamics for $\left[\mathrm{Fe}(\text { btbip })_{2}\right]^{2+}$ obtained by applying the correction in the interaction Hamiltonian, i.e., using the TDM vectors defined in Equation 5, for four polarization angles. As is seen from the panels, the excited-state populations are independent of the polarization. ${ }^{28}$

\section{Acknowledgement}

The research leading to the presented results has received funding from the People Programme (Marie Curie Actions) of the European Union's Seventh Framework Programme (FP7/2007-2013) under REA grant agreement no. 609405 (COFUNDPostdocDTU), and was also supported by the Government of Hungary and the European Regional Development Fund under grant VEKOP-2.3.2-16-2017-00015. 


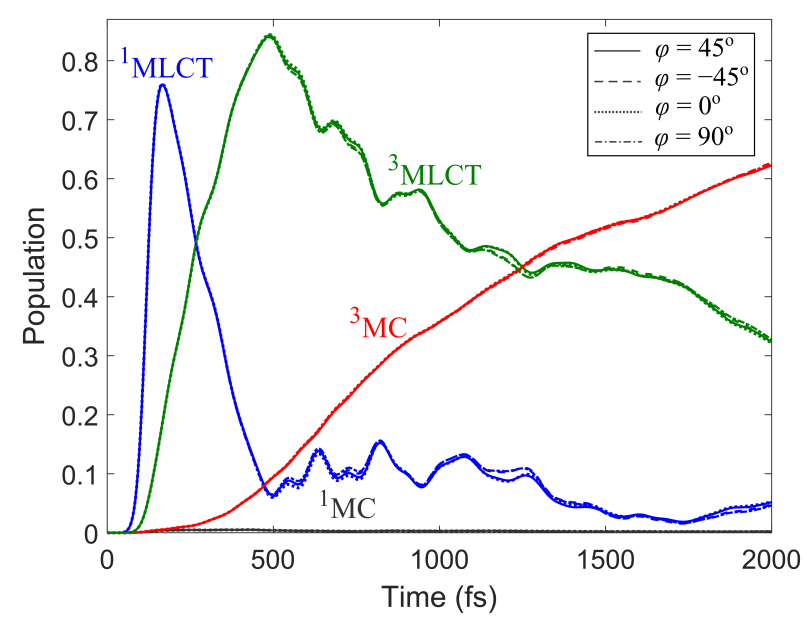

Figure 8: Diabatic population dynamics for $\left[\mathrm{Fe}(\mathrm{bmip})_{2}\right]^{2+}$ obtained by applying the correction in the interaction Hamiltonian, i.e., using the TDM vectors defined in Equation 5 , for four polarization angles. As is seen in the figure, the excited-state populations are independent of the polarization.

\section{References}

(1) Lasorne, B.; Worth, G. A.; Robb, M. A. Excited-State Dynamics. Wiley Interdiscip. Rev. Comput. Mol. Sci. 2011, 1, 460-475.

(2) Tavernelli, I. Nonadiabatic Molecular Dynamics Simulations: Synergies between Theory and Experiments. Acc. Chem. Res. 2015, 48, 792-800.

(3) Worth, G. A.; Meyer, H.-D.; Cederbaum, L. S. Relaxation of a System with a Conical Intersection Coupled to a Bath: A Benchmark 24-Dimensional Wave Packet Study Treating the Environment Explicitly. J. Chem. Phys. 1998, 109, 3518-3529.

(4) Woywod, C.; Domcke, W.; Sobolewski, A. L.; Werner, H.-J. Characterization of the S1S2 Conical Intersection in Pyrazine Using Ab Initio Multiconfiguration Self-ConsistentField and Multireference Configuration-Interaction Methods. J. Chem. Phys. 1994, 100, 1400-1413.

(5) Penfold, T. J.; Worth, G. A. A Model Hamiltonian to Simulate the Complex Photochemistry of Benzene II. J. Chem. Phys. 2009, 131, 064303. 
(6) Capano, G.; Chergui, M.; Rothlisberger, U.; Tavernelli, I.; Penfold, T. J. A Quantum Dynamics Study of the Ultrafast Relaxation in a Prototypical Cu(I)-Phenanthroline. J. Phys. Chem. A 2014, 118, 9861-9869.

(7) Eng, J.; Gourlaouen, C.; Gindensperger, E.; Daniel, C. Spin-Vibronic Quantum Dynamics for Ultrafast Excited-State Processes. Acc. Chem. Res. 2015, 48, 809-817.

(8) Pápai, M.; Vankó, G.; Rozgonyi, T.; Penfold, T. J. High-Efficiency Iron Photosensitizer Explained with Quantum Wavepacket Dynamics. J. Phys. Chem. Lett. 2016, 7, 20092014.

(9) Atkins, A. J.; González, L. Trajectory Surface-Hopping Dynamics Including Intersystem Crossing in $\left[\mathrm{Ru}(\mathrm{bpy})_{3}\right]^{2+}$. J. Phys. Chem. Lett. 2017, 8, 3840-3845.

(10) Capano, G.; Penfold, T. J.; Chergui, M.; Tavernelli, I. Photophysics of a Copper Phenanthroline Elucidated by Trajectory and Wavepacket-Based Quantum Dynamics: A Synergetic Approach. Phys. Chem. Chem. Phys. 2017, 19, 19590-19600.

(11) Harabuchi, Y.; Eng, J.; Gindensperger, E.; Taketsugu, T.; Maeda, S.; Daniel, C. Exploring the Mechanism of Ultrafast Intersystem Crossing in Rhenium(I) Carbonyl Bipyridine Halide Complexes: Key Vibrational Modes and Spin-Vibronic Quantum Dynamics. J. Chem. Theory Comput. 2016, 12, 2335-2345.

(12) Pápai, M.; Penfold, T. J.; Møller, K. B. Effect of tert-Butyl Functionalization on the Photoexcited Decay of a Fe(II)-N-Heterocyclic Carbene Complex. J. Phys. Chem. C 2016, 120, 17234-17241.

(13) Fumanal, M.; Gindensperger, E.; Daniel, C. Ultrafast Excited-State Decays in $\left[\operatorname{Re}(\mathrm{CO})_{3}(\mathrm{~N}, \mathrm{~N})(\mathrm{L})\right]^{n+}:$ Nonadiabatic Quantum Dynamics. J. Chem. Theory Comput. 2017, 13, 1293-1306. 
(14) Penfold, T. J.; Gindensperger, E.; Daniel, C.; Marian, C. M. SpinVibronic Mechanism for Intersystem Crossing. Chem. Rev. [Online early access]. DOI: 10.1021/acs.chemrev.7b00617. Published Online: Mar 20, 2018. https://pubs.acs.org/doi/10.1021/acs.chemrev.7b00617 (accessed Apr 17, 2018).

(15) Köppel, H.; Domcke, W.; Cederbaum, L. S. Advances in Chemical Physics, Volume LVII; John Wiley \& Sons, Inc., 1984; pp 59-246.

(16) Liu, Y.; Harlang, T.; Canton, S. E.; Chábera, P.; Suárez-Alcántara, K.; Fleckhaus, A.; Vithanage, D. A.; Göransson, E.; Corani, A.; Lomoth, R.; Sundström, V.; Wärnmark, K. Towards Longer-Lived Metal-to-Ligand Charge Transfer States of Iron(II) Complexes: An N-Heterocyclic Carbene Approach. Chem. Commun. 2013, $49,6412-6414$.

(17) Liu, Y.; Persson, P.; Sundström, V.; Wärnmark, K. Fe N-Heterocyclic Carbene Complexes as Promising Photosensitizers. Acc. Chem. Res. 2016, 49, 1477-1485.

(18) Only those TDM elements are computed and used in the Hamiltonian which correspond to excitation from the ground state, since transitions between excited states are far offresonant with respect to the exciting laser wavelength.

(19) Reiher, M.; Salomon, O.; Artur Hess, B. Reparameterization of Hybrid Functionals Based on Energy Differences of States of Different Multiplicity. Theor. Chem. Acc. $2001,10 \%, 48-55$.

(20) Gatti, F.; Lasorne, B.; Meyer, H.-D.; Nauts, A. Applications of Quantum Dynamics in Chemistry, Lecture Notes in Chemistry, vol 98; Springer International Publishing, 2017.

(21) Note that the TDM vectors to the degenerate singlet states as obtained from the electronic structure computations performed without symmetry constraints were originally 
not aligned to the $x$ and $y$ axes for both complexes. This is due to the fact that the degenerate states can be freely rotated among themselves in the Hilbert space. Therefore, the SOC elements given in refs. 8 and 12 were transformed according to a rotation of the pair of ${ }^{1} \mathrm{MLCT} /{ }^{1} \mathrm{MC}$ states by $182.77 / 88.2$ and 37.19/179.35 degrees for complexes $\mathbf{1}$ and $\mathbf{2}$, respectively, leading to TDMs lying on the axes, as illustrated in Figure 2.

(22) Meyer, H.-D.; Manthe, U.; Cederbaum, L. The Multi-Configurational Time-Dependent Hartree Approach. Chem. Phys. Lett. 1990, 165, $73-78$.

(23) Beck, M.; Jäckle, A.; Worth, G.; Meyer, H.-D. The Multiconfiguration Time-Dependent Hartree (MCTDH) Method: A Highly Efficient Algorithm for Propagating Wavepackets. Phys. Rep. 2000, 324, 1- 105.

(24) Meyer, H.-D.; Gatti, F.; Worth, G. Multidimensional Quantum Dynamics: MCTDH Theory and Applications; John Wiley and Sons, 2009; pp 1-419.

(25) Note that throughout this work we show diabatic populations. It is, however, important to state that the reported polarization dependence of the simulated dynamics arises in both the diabatic and adiabatic bases. This is confirmed by test simulations showing that adiabatic populations also exhibit strong polarization dependence.

(26) Siebrand, W.; Zgierski, M. Z. In Excited States; Lim, E. C., Ed.; Academic Press, 1979; Vol. 4; Chapter Resonance Raman Spectroscopy-A Key to Vibronic Coupling.

(27) Pawlikowski, M.; Pilch, M.; Mortensen, O. S. Vibronic Theory of Magnetic Vibrational Circular Dichroism in Systems with Fourfold Symmetry: Theoretical Analysis for Copper Tetraphenylporphyrin. J. Chem. Phys. 1992, 96, 4982-4990.

(28) The populations for $\left[\mathrm{Fe}(\text { btbip })_{2}\right]^{2+}$ shown in Figure 8 slightly differ from the results of our recent simulations, ${ }^{12}$ in which the wavepacket was impulsively excited to the ${ }^{1}$ MLCT states utilizing the $(+1 / \sqrt{2},+1 / \sqrt{2})$ weights (corresponding to the case of $\varphi=$ 
$\left.45^{\circ}\right)$. However, the excited-state lifetimes only vary by a factor of ca. 2. Consequently, the photorelaxation mechanism proposed and the conclusions drawn in ref. 12 are unaltered. 
For Table of Contents Only

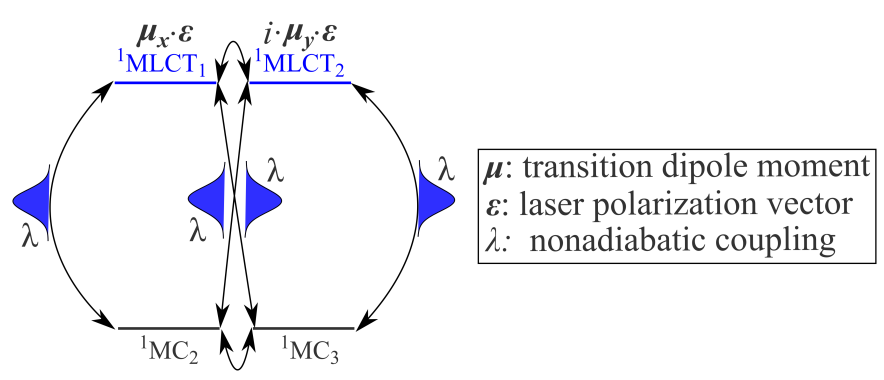



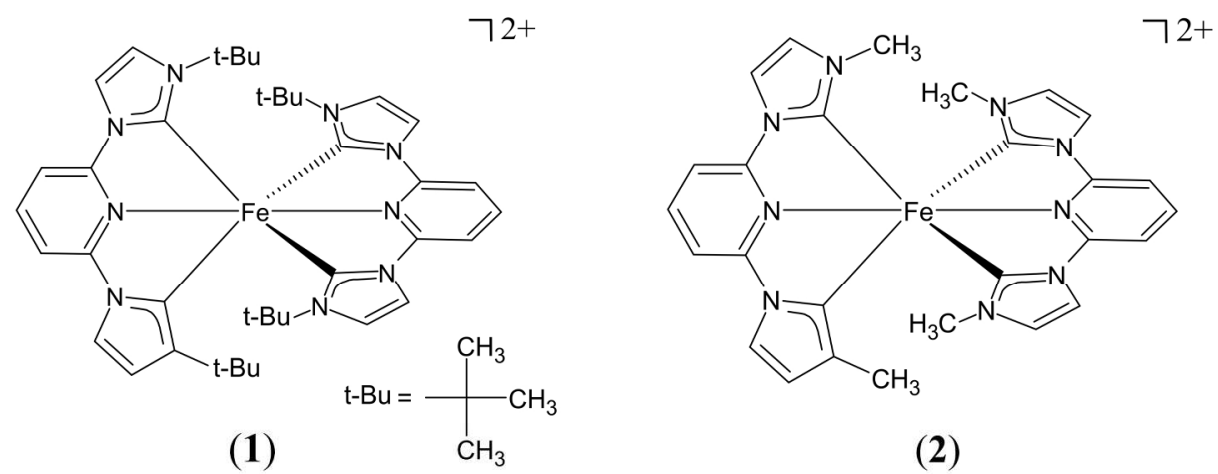

(2)
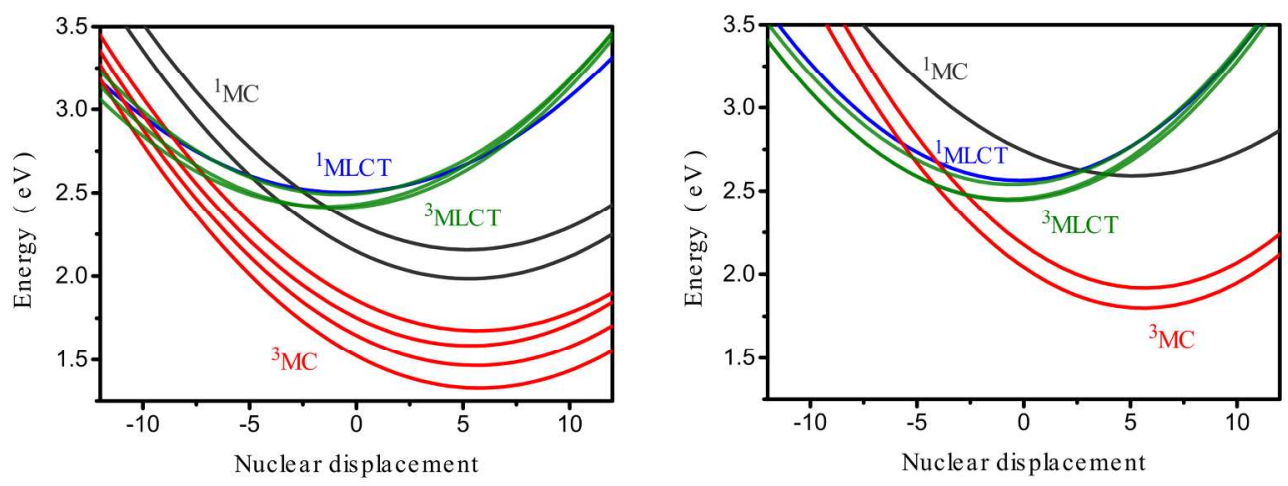

$203 \times 150 \mathrm{~mm}(300 \times 300$ DPI $)$ 


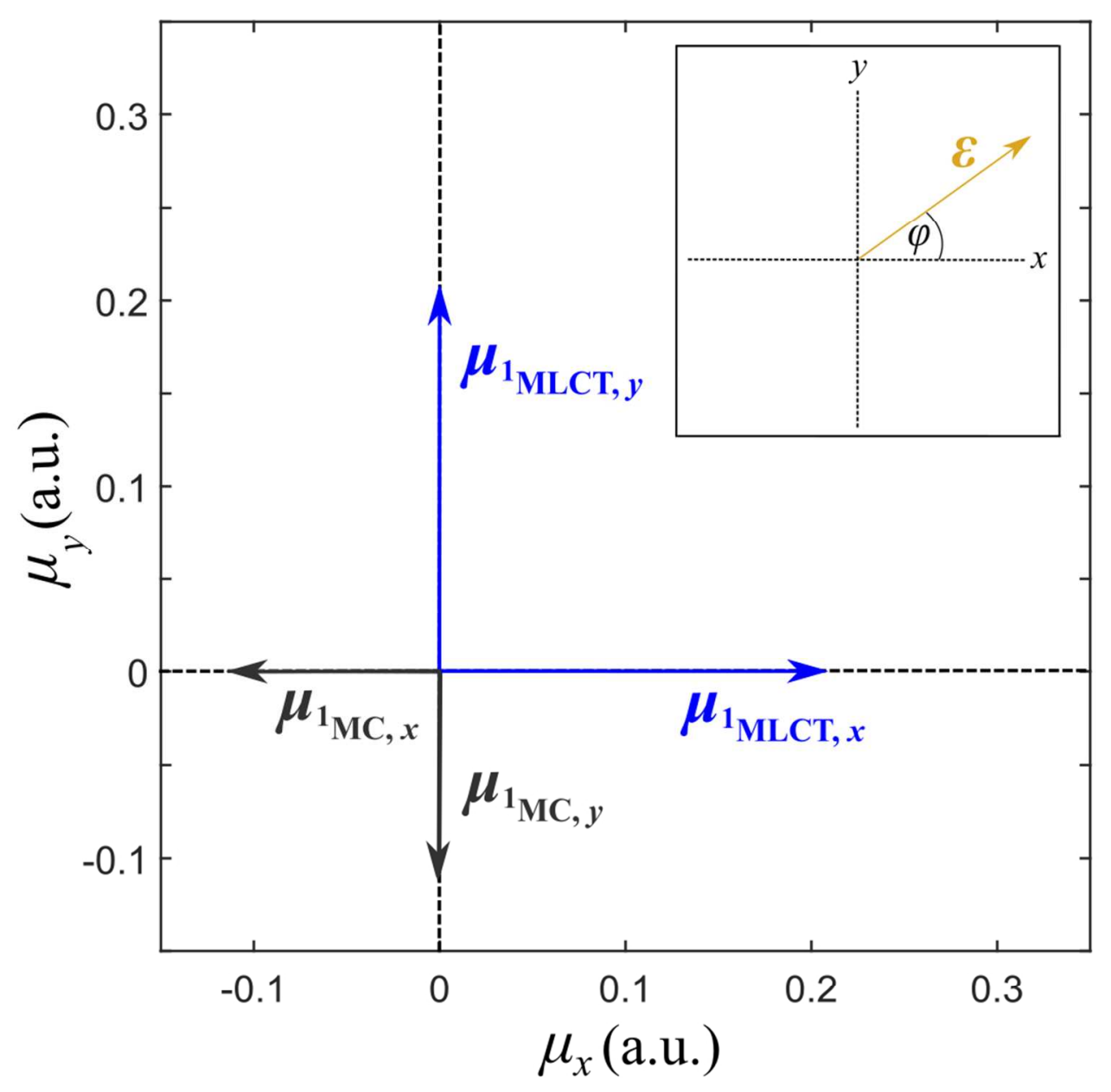

$92 \times 89 \mathrm{~mm}(300 \times 300 \mathrm{DPI})$ 

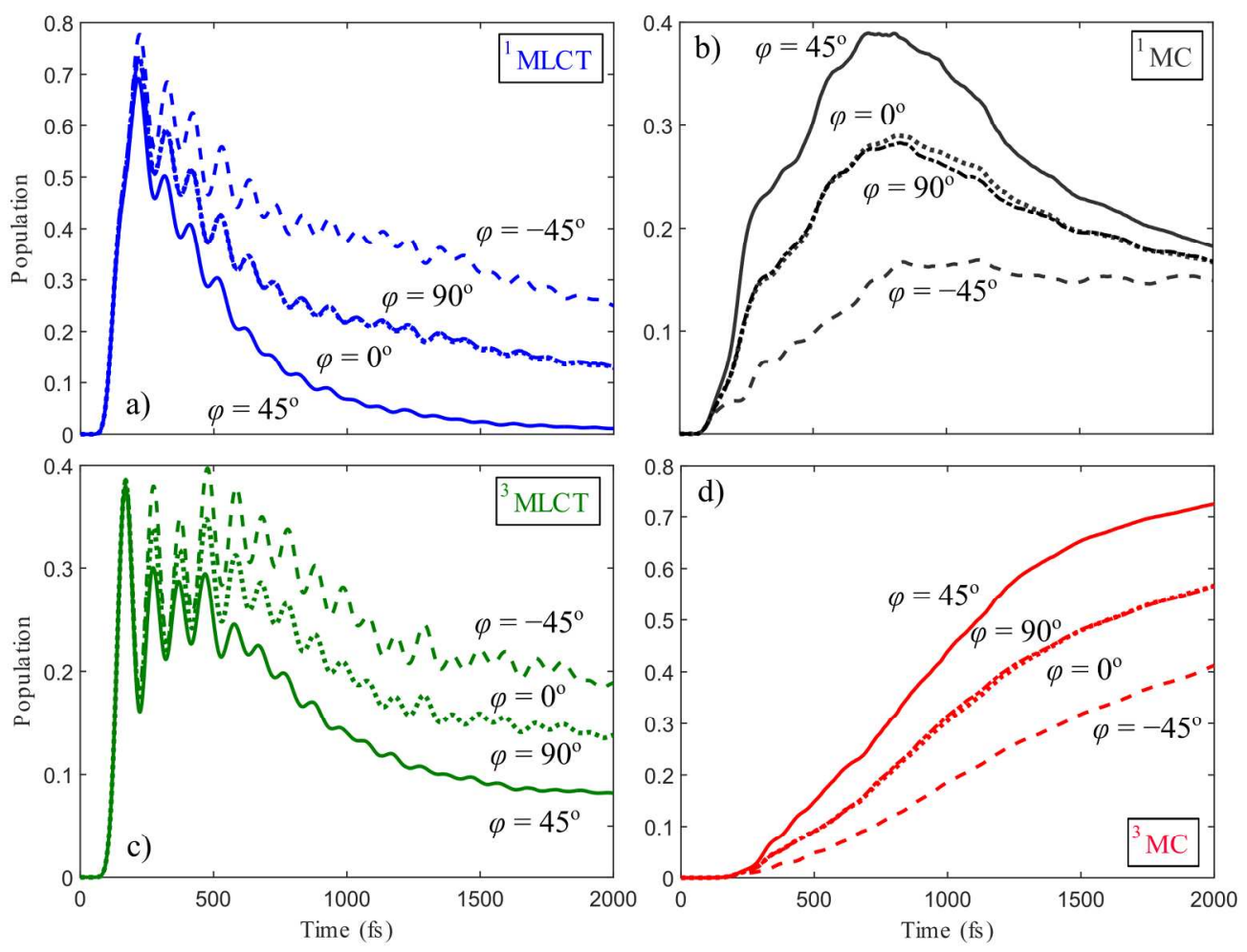

$183 \times 139 \mathrm{~mm}(300 \times 300$ DPI $)$ 


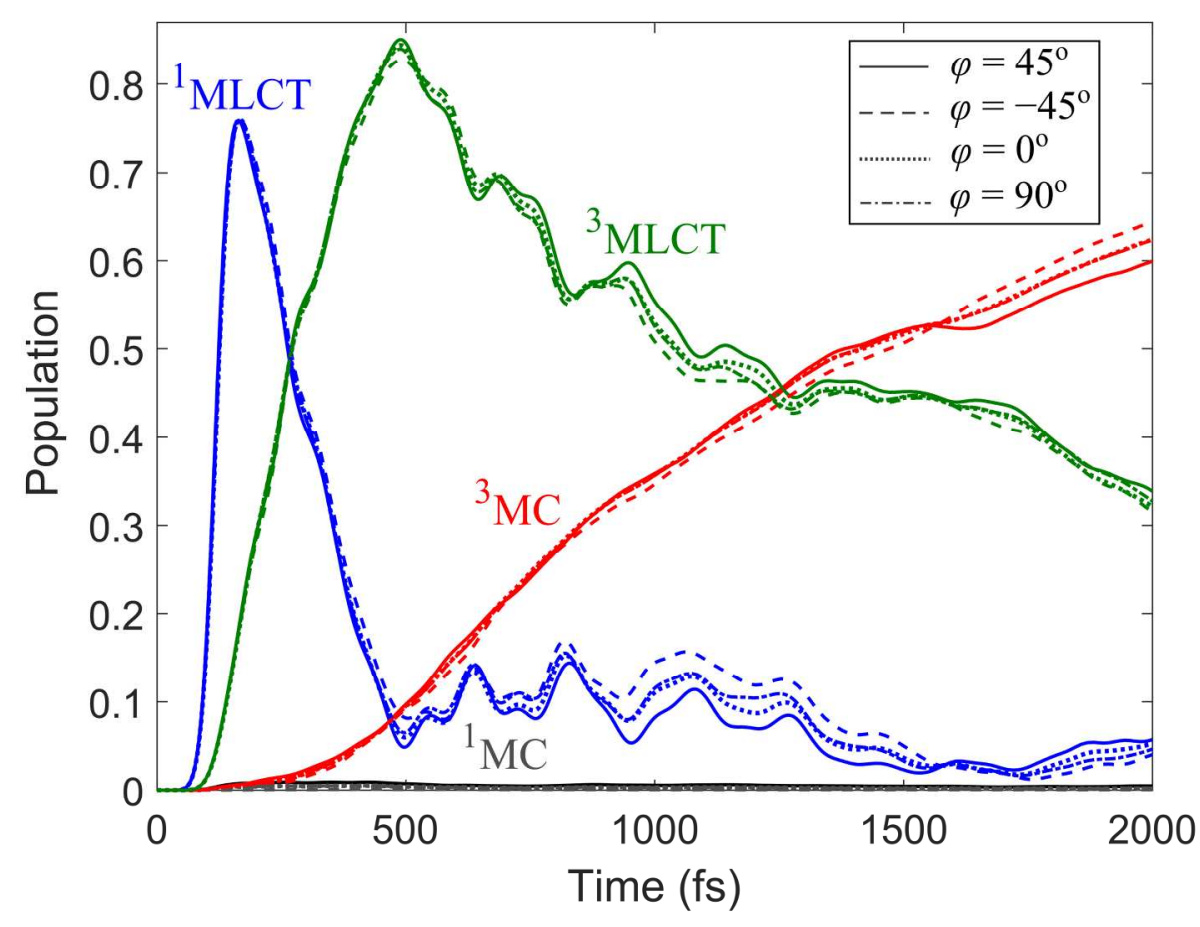

$205 \times 155 \mathrm{~mm}(300 \times 300$ DPI $)$ 
a) Nonadiabatic coupling (NAC)

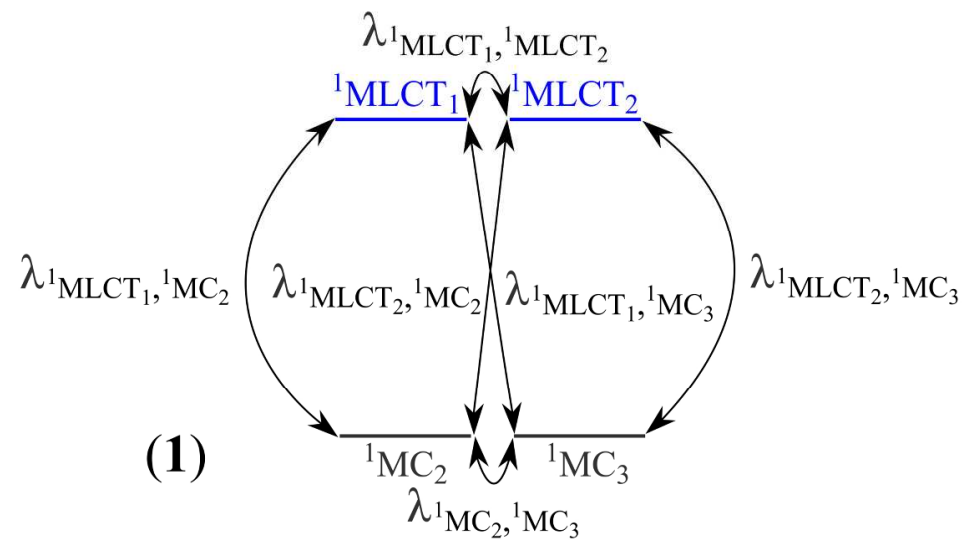

b) Spin-orbit coupling (SOC)
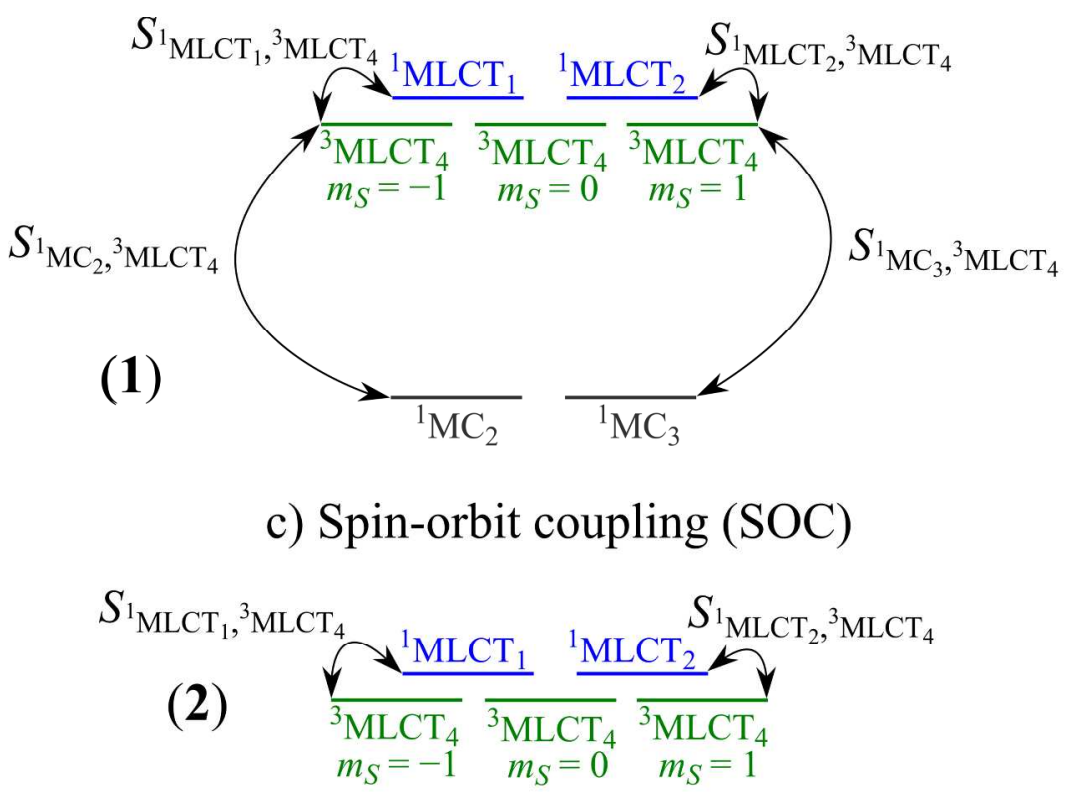

$181 \times 264 \mathrm{~mm}(300 \times 300 \mathrm{DPI})$ 


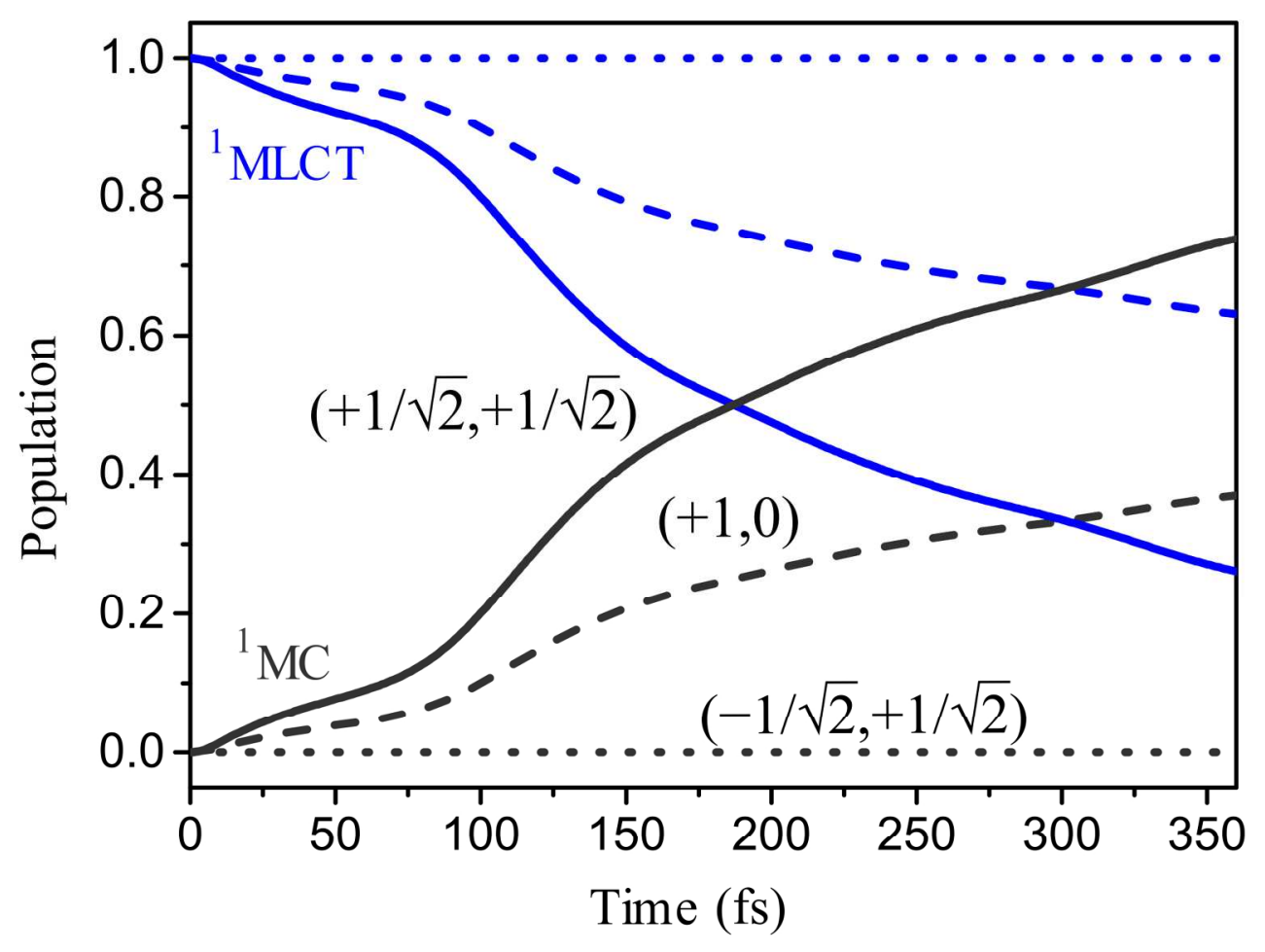

$174 \times 131 \mathrm{~mm}(300 \times 300$ DPI $)$ 

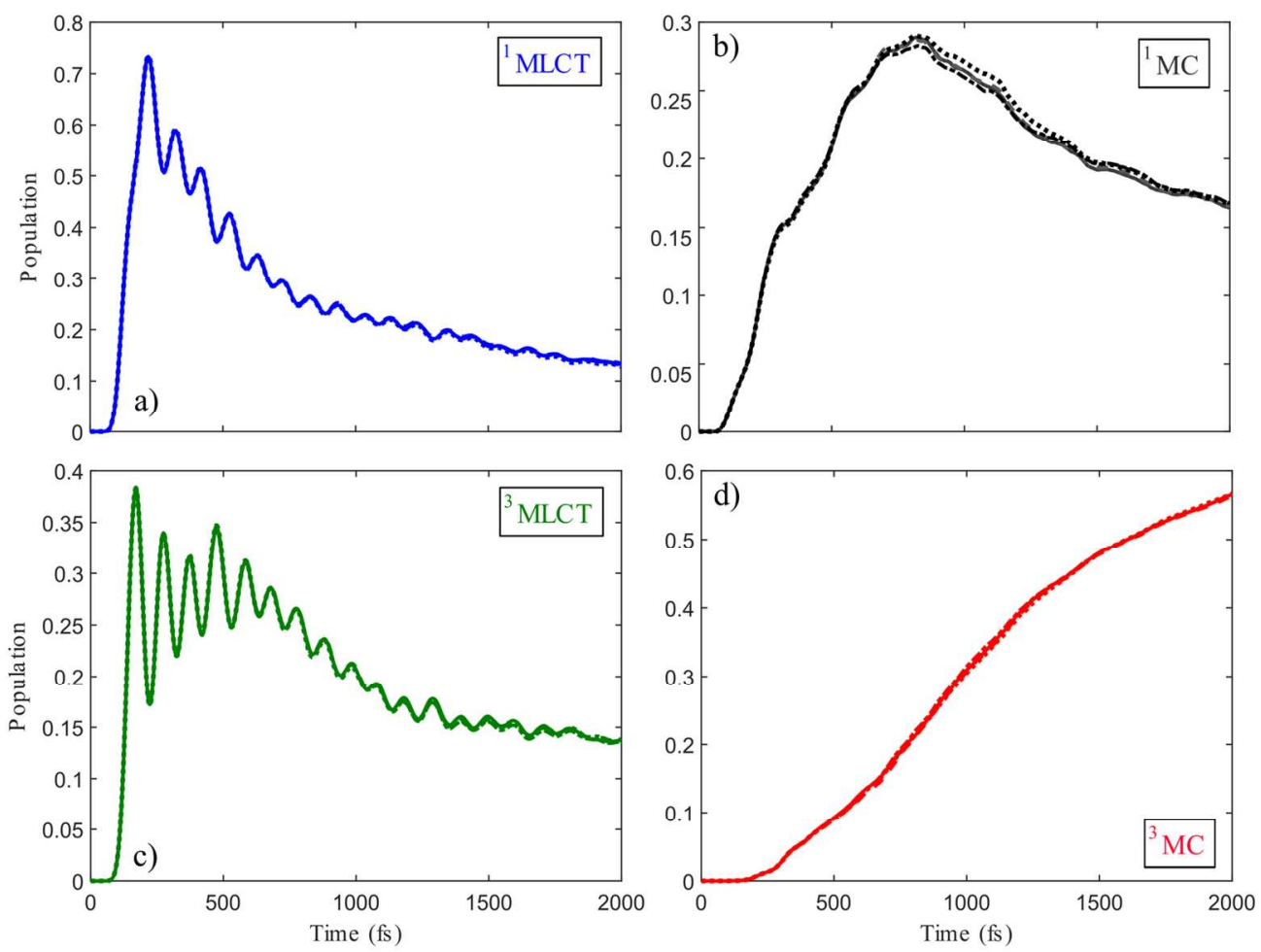

$140 \times 105 \mathrm{~mm}(300 \times 300$ DPI $)$ 


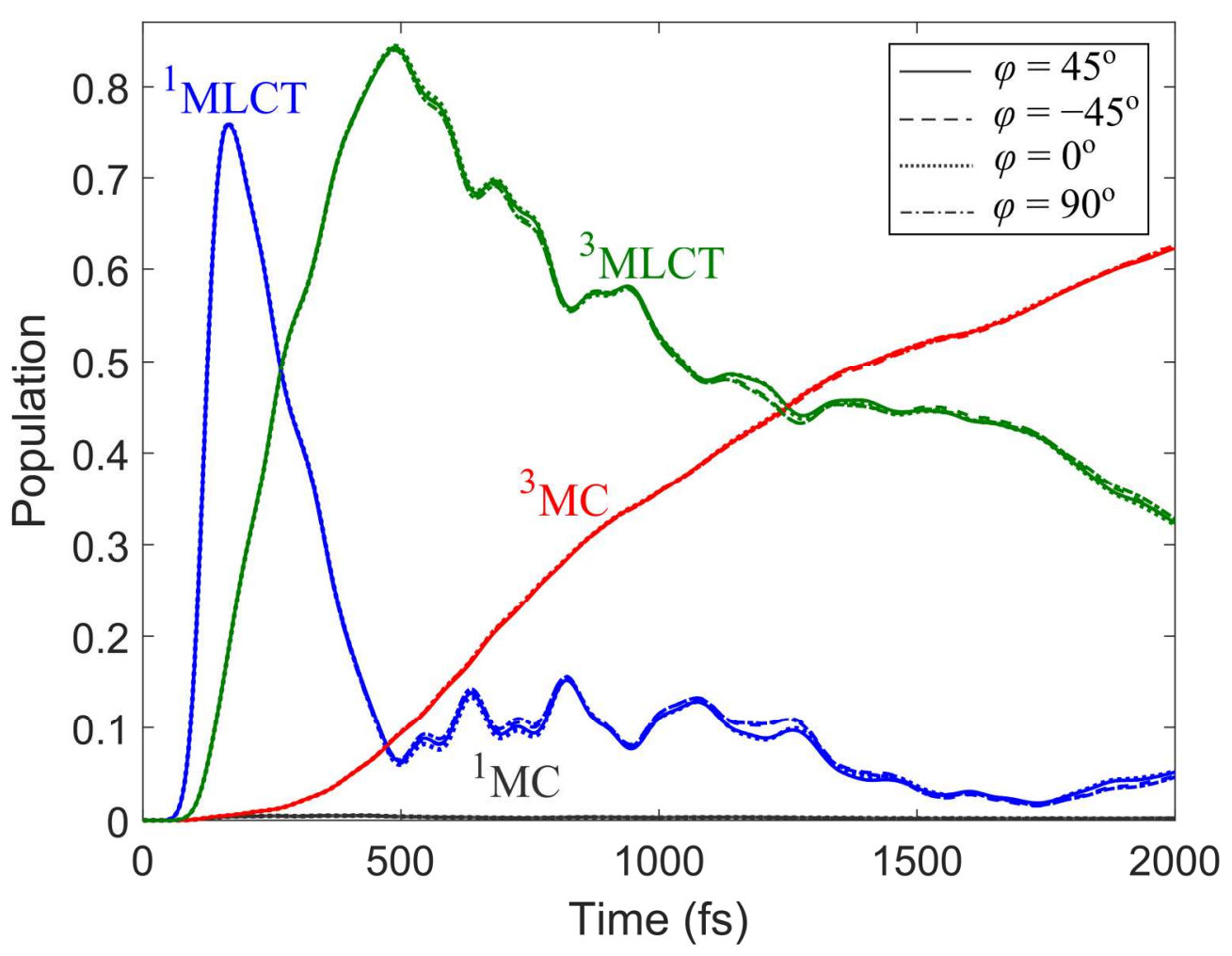

$187 \times 143 \mathrm{~mm}(300 \times 300$ DPI $)$ 


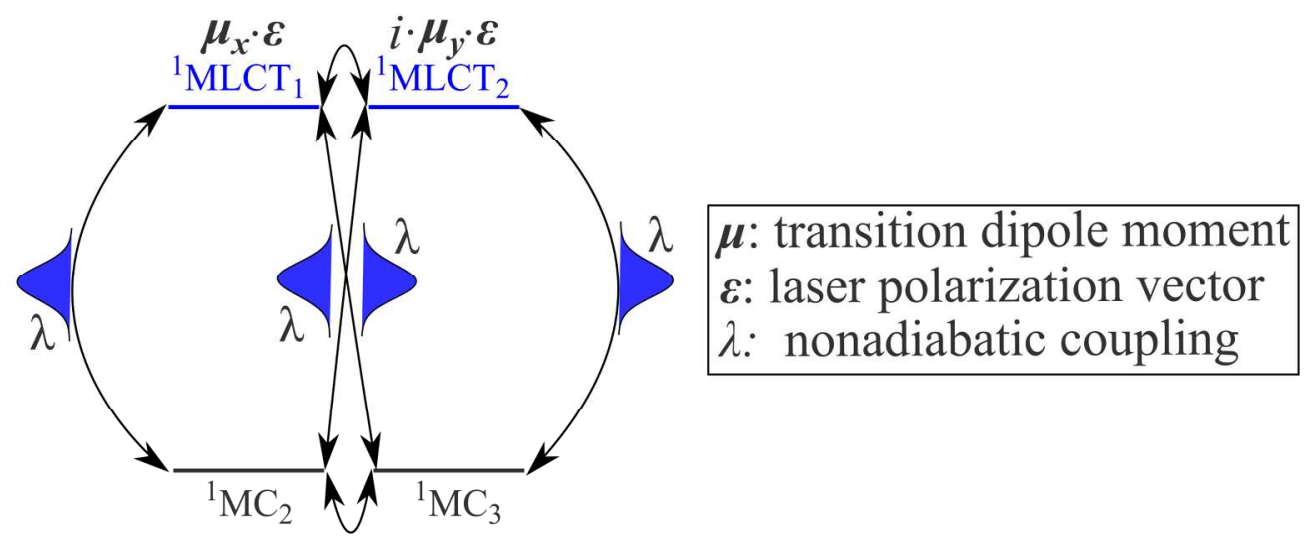

$191 \times 78 m m(300 \times 300$ DPI $)$ 